\title{
ASI outreach
}

The outreach component of the AstroSat mission started even before its launch and continued well into 2019. Prior to launch, a booklet was written that explained the rationale behind AstroSat, its various payloads, and its science case, aimed at college students. Multi-lingual FAQs accompanied this effort. In the week of the launch, press conferences were organised in three different cities. In the following months, a set of well-designed posters were created and printed that described the key features of a Space Observatory. The longest running outreach program was AstroSat Picture of the Month (APOM) (https://astron-soc.in/outreach/apom/), which featured a monthly image from AstroSat accompanied by explanatory text and links for further reading. Many of these APOMs were also recast as articles that were published in many newspapers. All of these resources reached the student and teacher communities in India through social media, some of which were dedicated to AstroSat. These activities were organised by the Public Outreach and Education Committee of the Astronomical Society of India in collaboration with the AstroSat Training and Outreach Team. 